\title{
Innovation, Ungleichheit und sozialer Aufstieg
}

\section{Verena Maria Konzett}

\section{Relevanz}

Innovation treibt das Wachstum, aber wenige erfolgreiche Unternehmer, Ingenieure und Forscher profitieren überdurchschnittlich stark. Gerade die erfolgreichsten Innovationen schaffen ungeahnte Vermögen und tragen zur Konzentration der Spitzeneinkommen bei. Gleichzeitig ist innovatives Unternehmertum eine grosse Chance für sozialen Aufstieg und verhindert eine Zementierung der Ungleichheit. Der Wettbewerb durch neue innovative Unternehmen fordert die etablierten Konzerne heraus und verhindert unverdiente Renteneinkommen. Innovative Unternehmensgründungen und die Beseitigung von Marktzutritts- und Wettbewerbsbarrieren fördern inklusives Wachstum, indem sie unproduktiven Vermögenskonzentrationen entgegenwirken.

\section{Quelle}

Aghion, Philippe, Ufuk Akcigit, Antonin Bergeaud, Richard Blundell und David Hemous (2019), Innovation and Top Income Inequality, Review of Economic Studies 86, 1-45.

Die Einkommensungleichheit hat während der letzten Jahrzehnte in den Industrieländern stark zugenommen. Doch bis heute konnte noch kein Konsens darüber erzielt werden, welche Faktoren hauptverantwortlich für diesen Anstieg sind. Ein Grund könnte in der immer wichtiger werdenden Rolle von Innovation in ent-

V. M. Konzett $(\square)$

Universität St.Gallen, St.Gallen, Schweiz

E-Mail: verena.konzett@student.unisg.ch 
wickelten Volkswirtschaften liegen. Denn die Erträge von Innovationen sind stark konzentriert. Sie fallen zu einem grossen Teil wenigen Wissenschaftlern, Unternehmern oder Ingenieuren zu und steigern dadurch die Spitzeneinkommen überproportional. Das kann letztlich zu einer grösseren Einkommensungleichheit führen.

Ein Forscherteam um Philippe Aghion und Richard Blundell untersucht den Zusammenhang zwischen Innovation, Konzentration der Spitzeneinkommen und sozialer Mobilität in den USA. Die beschreibende Evidenz in Abb. 1 deutet auf einen positiven Zusammenhang zwischen der Innovationstätigkeit, gemessen an der Anzahl zitierter Patente, und dem Anteil der Spitzeneinkommen in den amerikanischen Bundesstaaten zwischen 1980 und 2005 hin. Zudem nahm die Ungleichheit besonders in jenen Berufen zu, die eng mit der Innovationstätigkeit zusammenhängen, wie Ingenieure, Unternehmer, Wissenschaftler und Manager.

Die Wissenschaftler entwickeln zuerst ein theoretisches Modell in der Tradition Joseph Schumpeters und seiner Idee der kreativen Zerstörung, um damit die wichtigsten Zusammenhänge zwischen Innovation, Ungleichheit und sozialer Mobilität zu erklären. Der Ansatz betont qualitätssteigernde Innovationen sowohl durch neu eintretende als auch durch bereits etablierte Unternehmen als zentralen Treiber wirtschaftlichen Wachstums. Daraus leiten sie drei Hypothesen $a b$, die sie empirisch überprüfen: Erstens erhöhen Innovationen die Spitzenein-

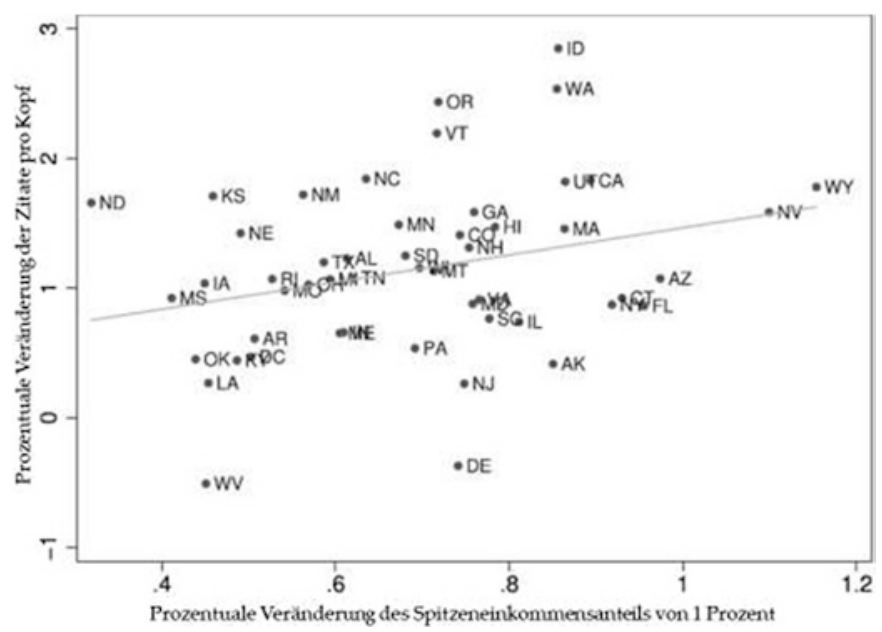

Abb. 1 Prozentuale Zunahme des Anteils der Top $1 \%$ Einkommen in US-Bundesstaaten. (Quelle: Aghion u. a. 2019) 
kommen überdurchschnittlich stark, wodurch die Einkommensungleichheit zunimmt. Zweitens verbessern Innovationen durch neu eintretende Unternehmen die soziale Mobilität, d. h. mehr Innovationen fördern den sozialen Aufstieg erfolgreicher Persönlichkeiten und greifen die Besitzstände der Etablierten an. Und drittens, eine Behinderung des Wettbewerbs durch Marktzutrittsbarrieren hemmt die soziale Mobilität und verringert damit auch den Einfluss von Innovationen durch neue Unternehmen auf die Ungleichheit.

Die Wissenschaftler überprüfen und quantifizieren diese Hypothesen mithilfe von Daten zur Einkommensverteilung und Innovationstätigkeit in den U.S. Bundesstaaten zwischen 1976 und 2009. Neben anderen Massen für Ungleichheit betrachten sie vorwiegend den Anteil der Top $1 \%$ Verdiener am Gesamteinkommen des jeweiligen Bundesstaats. Die Anzahl von Patenten pro Kopf und Jahr misst den Umfang der Innovationstätigkeit. Dies ist lediglich ein grobes Mass, da es grosse Qualitätsunterschiede zwischen Patenten gibt. Daher berechnen sie Messgrössen für qualitätsangepasste Innovationen, indem sie beispielsweise jene Patente, die oft von anderen zitiert werden, stärker gewichten. Zudem berücksichtigen die Forscher weitere Faktoren, welche Innovationen und Spitzeneinkommen ebenfalls beeinflussen können, wie etwa die Beschäftigung im Finanzsektor, die Grösse des Staatssektors, die durchschnittlichen Einkommen oder die Steuerbelastung.

Innovation und Einkommensanteil der Top $1 \%$ Verdiener sind signifikant positiv korreliert. Der Zusammenhang zwischen Innovation und Einkommensverteilung über die gesamte Bevölkerung ist hingegen schwächer ausgeprägt.

Die empirischen Schätzungen deuten auf einen positiven Zusammenhang hin. Mehr Innovationen steigern den Einkommensanteil der Top $1 \%$ der Spitzenverdiener unabhängig davon, wie genau Innovationen gemessen werden. Der Einfluss der qualitätsangepassten Patentzahl ist tendenziell grösser. Öfter zitierte Patente stellen eher jene echten Innovationen dar, welche besonders hohe Erträge abwerfen. Der Zusammenhang zwischen der Innovationsintensität und den umfassenderen Ungleichheitskennzahlen, wie z. B. dem oft verwendeten Gini-Koeffizienten, ist hingegen kaum signifikant oder sogar leicht negativ. Eine höhere Innovationsintensität verschärft die Ungleichheit vor allem dadurch, dass der Anteil der Top $1 \%$ der Spitzeneinkommen überproportional stark steigt.

Zudem weisen die Forscher darauf hin, dass Innovationen sowohl durch neu eintretende als auch durch bereits etablierte Unternehmen positiv mit der Einkommensungleichheit zusammenhängen. Wenn jedoch die etablierten Unternehmen etwa durch Lobbying versuchen, den Zutritt neuer Konkurrenten 
zu erschweren, dann hat die Innovation durch neu eintretende Firmen eine schwächere Auswirkung auf die Einkommensungleichheit.

Im Durchschnitt aller Staaten und im Zeitraum zwischen 1980 und 2005 erklärt die Zunahme der Innovationen etwa 23 Prozent des Gesamtanstiegs im Einkommensanteil der Top $1 \%$ Verdiener.

Die Forscher schätzen, dass ein Anstieg in der Anzahl der Patente pro Kopf um $1 \%$ den Einkommensanteil der Top $1 \%$ Verdiener um $0.22 \%$ erhöht. Berücksichtigt man die Qualitätsunterschiede bei Patenten, ergibt sich ein ähnlicher Effekt. Zum Beispiel hat sich in Kalifornien zwischen 1980 und 2005 der Anteil der Spitzeneinkommen mehr als verdoppelt. Die empirischen Ergebnisse bedeuten, dass die Zunahme der Einkommenskonzentration zu etwa $29 \%$ auf das Konto zunehmender Innovation geht. Im Durchschnitt aller Bundesstaaten erklären steigende Innovationen etwa $23 \%$ des Anstiegs der Einkommensungleichheit. Dennoch unterschätzt die Analyse tendenziell die wahren Auswirkungen von Innovationen auf die Ungleichheit unter den Spitzeneinkommen. So können beispielsweise Erfinder in einen anderen Bundesstaat übersiedeln, wodurch die Innovationen dem einen, die Erträge daraus aber einem anderen Bundesstaat zugeordnet werden.

Innovation verbessert die Aufstiegschancen. Steigt die Anzahl der Patente um das 2.5-Fache, nimmt die Wahrscheinlichkeit, zu den $20 \%$ der höchsten Einkommen aufzusteigen, um über $10 \% \mathrm{zu}$.

Wie wirken sich Innovationen auf die soziale Mobilität und damit auf die Aufstiegschancen in der Gesellschaft aus? Dazu betrachten die Forscher die Wahrscheinlichkeit, im Alter von 30 Jahren ein Einkommen zu erzielen, das zu den höchsten $20 \%$ zählt, wenn das Einkommen der Eltern zu den untersten $20 \%$ zählte. Diese Aufstiegswahrscheinlichkeit beträgt durchschnittlich $9.6 \%$. Innovation kann die Aufstiegschancen signifikant verbessern. So steigt die Aufstiegswahrscheinlichkeit um 1.2 Prozentpunkte, wenn die Anzahl der Patente pro Kopf um das 2.5-Fache zunimmt, das heisst, sich mehr als verdoppelt. Allerdings haben nur Innovationen neu eintretender Unternehmen einen solchen Effekt, während Innovationen bestehender Firmen die soziale Mobilität nicht signifikant erhöhen. Im Gegenteil, wenn bestehende Firmen, z. B. mit Lobbying, Marktzutrittsbarrieren errichten und den Wettbewerb durch neue Konkurrenten behindern, dann verringern sie die Aufstiegschancen mittels innovativer Unternehmensgründungen. 
Die Erträge erfolgreicher Innovationen fallen überwiegend wenigen Haushalten und Personen zu. Dadurch steigt der Anteil der Spitzenverdiener am Gesamteinkommen und die Ungleichheit nimmt zu. Gleichzeitig verbessern innovative Unternehmensgründungen, welche die etablierten Unternehmen herausfordern und bestehende Besitzstände im Sinne „kreativer Zerstörung“ nach Schumpeter angreifen, die Aufstiegschancen in der Gesellschaft.

Open Access Dieses Kapitel wird unter der Creative Commons Namensnennung 4.0 International Lizenz (http://creativecommons.org/licenses/by/4.0/deed.de) veröffentlicht, welche die Nutzung, Vervielfältigung, Bearbeitung, Verbreitung und Wiedergabe in jeglichem Medium und Format erlaubt, sofern Sie den/die ursprünglichen Autor(en) und die Quelle ordnungsgemäß nennen, einen Link zur Creative Commons Lizenz beifügen und angeben, ob Änderungen vorgenommen wurden.

Die in diesem Kapitel enthaltenen Bilder und sonstiges Drittmaterial unterliegen ebenfalls der genannten Creative Commons Lizenz, sofern sich aus der Abbildungslegende nichts anderes ergibt. Sofern das betreffende Material nicht unter der genannten Creative Commons Lizenz steht und die betreffende Handlung nicht nach gesetzlichen Vorschriften erlaubt ist, ist für die oben aufgeführten Weiterverwendungen des Materials die Einwilligung des jeweiligen Rechteinhabers einzuholen.

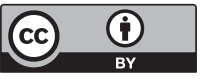

